\title{
Geographie und Entwicklungszusammenarbeit
}

\section{Grundlagen}

Es scheint, daß wir mit dem Verständnis der Begriffe Geographie und Entwicklungszusammenarbeit keine Mühe haben. Werden wir aber nach dem Sinn z. B. der Geographie gefragt, so ist es wichtig, auf gewisse Definitionswandel hinzuweisen, die diese Wissenschaft im letzten Jahrhundert durchgemacht hat. Von der eigentlichen Beschreibung der Erde, der Kartierung und Namengebung ihrer raumzeitlichen Kontinua, ist der Begriffsinhalt in die heute vielgeschmähte Erdkunde des Beginns unseres Jahrhunderts gewandert. Die sich daraus entwickelnde Länderkunde wurde als eine besonders auch auf Didaktik ausgelegte, streng gegliederte Beschreibung des ein-einheitlichen nationalen Raumes verstanden. $\mathrm{Da}$ diese Art der Umschreibung nicht geeignet war, regional-konzeptionelle, oft grenzüberschreitende sozio-ethnologische, ökologische oder naturräumliche Gliederungen zuzulassen, leuchtet ein. Ganz klar werden diese Mängel an sozialräumlichen Fragen der Anthropogeographie oder Humangeographie. Es bedurfte der Einflüsse der Nachbarnaturwissenschaften ebenso wie derjenigen der Geisteswissenschaften, um eine neue Geographie, die Geographie als Wissenschaft zur Erkennung und Beschreibung sowie Erfassung und Erklärung räumlicher Organisationsformen und Prozesse zu verstehen. Eine Geographie, die den Menschen in den Mittelpunkt rückt und den Geographen, ausgerüstet mit modernstem Rüstzeug der Natur- und Geisteswissenschaften, Stellung nehmen läßt zu aktuellen Problemen.

Das heißt, daß sich der Geograph je nach Aufgabenstellung sehr stark mit dem Fachwissen von Nachbarwissenschaften zu befassen hat. Sehr oft verschwinden dadurch die eigentlichen Grenzen, und man wäre geneigt zu sagen, daß je nach Sachlage ebensogut ein Soziologe, Ethnologe, Historiker, Ökonom, Physiker, Geologe, Biologe oder Ökologe sich mit dem Problem auseinandersetzen könnte. Wo liegt nun aber das spezifisch Geographische, welches den Geographen so sehr geeignet erscheinen läßt, in der Entwicklungszusammenarbeit tätig zu sein? Der unterschiedliche Blickpunkt - beim Geographen der Raumbezug - die Suche nach raumzeitlichen Organisationen, organisierten Formen und
Prozessen ermöglicht ihm sehr oft geeignete Teilbereiche aus Einzelwissenschaften zusammenzufassen und in gezielten Anwendungen in einen sinnvollen Zusammenhang zu bringen. Letztlich wird die Leistung einer Wissenschaft gemessen an der Möglichkeit, zu aktuellen Problemen konstruktiv Stellung nehmen und brauchbare Lösungen anbieten zu können.

Was die Geographie ist und will, scheint nun klarer zu werden. Wenden wir uns darum dem zweiten Begriff zu, der Entwicklungszusammenarbeit, bevor wir die Möglichkeiten der Geographie in diesem Aufgabenkreis an einigen praktischen Beispielen aufzuzeigen versuchen.

Das Wort Entwicklungszusammenarbeit selbst zeigt differenziert seine Zweckbestimmung. Der Begriff der Entwicklungshilfe dagegen geht davon aus, daß jemand sich in der Lage sieht oder auch darin ist, einem anderen, der sich in einem potentiellen Entwicklungsnotstand befindet, zu helfen. Es bedarf, daß Entwicklungshilfe geleistet wird, offenbar eines Gefälles zwischen Donator und Rezeptor. Je nach Lage in diesem System kann bereits diese Tatsache erfreulich für den einen, aber auch frustrierend für den anderen sein. Diese Hilfe in einem bestimmten entwicklungsfähigen Sektor heißt eben nicht eine generelle Überlegenheit des einen über den andern oder gar eine Systemüberlegenheit. Ganz klar sprechen wir hier nicht von Katastrophenhilfe oder anderer humanitärer Hilfe, die ein menschliches Gebot ist, sondern von der Hilfe zur Selbsthilfe durch die sogenannte Entwicklungszusammenarbeit. Es handelt sich hierbei um eine Hilfe unter Gleichberechtigten, unter Partnern.

Das Eidgenössische Departement für auswärtige Angelegenheiten bringt diesen subtilen aber sehr wichtigen Unterschied im Namen der DEH, der Direktion für Entwicklungszusammenarbeit und humanitäre Hilfe, gut zum Ausdruck.

Ziel der eidgenössischen Entwicklungszusammenarbeit ist: «die dauernde Beseitigung der strukturellen Aspekte der Unterentwicklung durch eine Veränderung der wirtschaftlichen und sozialen Bedingungen unter den sich ergänzenden Grundsätzen der wirt-

PD Dr. Klaus I. Itten. Geographisches Institut der Universität Zürich, Postfach, 8033 Zürich. 
schaftlichen Interpendenz und der Solidarität.» (nach JOLLES, Lit. 1 u. EDA, Lit. 2).

Darin finden wir die drei Instrumente der schweizerischen Entwicklungszusammenarbeit:

1. Die technische Zusammenarbeit sowie bilaterale und multilaterale Finanzhilfe.

2. Die wirtschafts- und handelspolitischen Maßnahmen.

3. Die humanitäre Hilfe.

Altmeister der Geographie CARL TROLL wies in seiner vielbeachteten Rektoratsfestrede (Lit. 3) vor 20 Jahren darauf hin, daß Entwicklungsländer und ihre Probleme immer vor ihrem kultur- und sozialgeographischen Hintergrund differenziert betrachtet werden müssen. Er sieht in der vergleichenden Beurteilung und Zusammenschau der naturräumlichen, kulturellen, wirtschaftlichen und sozialen Differenzierung der betreffenden Gebiete - in dem was man heute im Hinblick auf die Planung der Entwicklungshilfe als die Erfassung der Infrastruktur bezeichnet - den Einsatz und die Möglichkeiten der Geographie. Dies soll und kann aber nicht allein durch den Geographen gelöst werden. Vielmehr braucht es das interdisziplinäre Zusammenwirken von Agronomen, Hydrologen, Ökologen, Ethnologen, Soziologen, Wirtschaftswissenschaftern, Ingenieuren und Technikern. Die generellen Problembetrachtungen bleiben aber in einem geographischethnographischen Ansatz verwurzelt.

Weiter führt TROLL aus, daß zur Analyse eines Entwicklungslandes historische Quellenstudien und demographische und volkswirtschaftliche Analysen basierend auf statistischen Erhebungen gehören, daß aber vor allem Feldforschungen den Experten in die Lage versetzen, das natur- und kulturbedingte Wirkungsgefüge eines Landes und seiner Teile aufzuklären.

Verlassen wir nun diese Gedanken TROLLs und versuchen, anhand einiger Beispiele aufzuzeigen, in welcher Weise man sich praktische Anwendungsmöglichkeiten vorzustellen hat. Es ist schwierig, aus der enormen Fülle von begeisternden Projekten eine Auswahl zu treffen. Gewisse Aspekte sollen hier systematisch gezeigt werden, wohl wissend, daß damit das Ausmaß der Möglichkeiten in seiner Breite nicht erschöpfend behandelt ist.

Die Aussage, daß es kaum mehr sogenannt «weiße Flecken» auf Landkarten und damit gänzlich unbekannte Gebiete gebe, stimmt zwar. Beschäftigen wir uns aber mit den grundlegenden Kenntnissen über Regionen in der dritten Welt, so sind wir oft erstaunt, wie spärlich dieses Wissen und diese vorhandenen Grunddaten sind.

1972 waren erst $35 \%$ der Erde in einem Maßstab von 1: 100000 oder besser kartiert (DOYLE, Lit. 4). Die jährliche Zuwachsrate beträgt $0,35 \%$. Das heißt, $\mathrm{da} ß$ in sehr vielen Fällen die Planung fast unmöglich ist, weil einfachste Kenngrößen zwar oft auf nationaler Ebene grob geschätzt, aber in ihrer regionalen Prägung und räumlichen Verbreitung nicht erfaßt sind.

Eine erste Aufgabe besteht darum oft in der Grunddatenbeschaffung, statistischen Bearbeitung und Darstellung auf einer meist ebenfalls erst herzustellenden Kartenbasis.

Als Beispiele für Grunddatenbeschaffungen betrachten wir konkrete Projekte von Geographen in Nordjemen, in Nepal, in Liberia und in Sri Lanka. Dann ist aber erst die Basis geschaffen für die Ermittlung von Systemdaten, die an den gleichen Projektbeispielen zum Teil ebenfalls aufgezeigt werden. Der Einsatz der Geographie in der Entwicklungszusammenarbeit kann unterteilt werden in:

1. Die Beschaffung von Grunddaten, insbesondere in die Erfassung

- der naturräumlichen Gliederung;

- der natürlichen Hilfsgüter oder Ressourcen;

- der Demographie, der Bevölkerung.

2. Die Beschaffung von Systemdaten über

- die kulturelle Gliederung;

- den arbeitenden Menschen;

- die Strukturen von Gesellschaft und Wirtschaft

- und die Systeme in bezug auf Entwicklungsplanung.

Einige der demonstrierten Projekte sind gegenwärtig noch im Gange, andere seit längerer Zeit abgeschlossen, doch so interessant, daß sie gut in diese Systematik passen.

\section{Das Beispiel Jemen}

In der Arabischen Republik Jemen wurde 1975 der erste Bevölkerungszensus durchgeführt. Nach dem Ersuchen der jemenitischen Regierung an unsere Behörden wurde ein schweizerisches Team von Geographen und einem Architekten unter der Leitung von STEFFEN beauftragt, die Genauigkeit der Bevölkerungszählung einerseits zu überprüfen, dann aber auch zu vervollständigen für Gebiete, in die keine jemenitischen Zähler hineingelangt waren, und dann die Daten statistisch und administrativ zu bearbeiten und zu"gliedern.

Als Grundlagenmaterial dienten Luftbilder sowie kleinmaßstäbliche englische Übersichtskarten $1: 250000$. Zunächst mußten Verwaltungsregionen, Zählbezirke und Siedlungen größenmäßig und kartographisch erfaßt werden. Durch Haustypenbestimmungen, Hauszählungen und Hochrechnungen mit durchschnittlichen Belegungsziffern, der Anzahl Bewohner pro Haustyp, galt es, die offiziellen Zählungen zu überprüfen. Die wichtigsten Haustypen wurden darum vermessen und demographisch 
erfaßt. Die statistisch bereinigten Zahlen wurden daraufhin multipliziert mit der Anzahl der betreffenden Häuser, die man mit Luftbildinterpretation ermittelt hatte und ergaben damit Einwohnerzahlen. Die ersten Resultate zeigten, daß ganz offensichtlich $z$. T. Siedlungen ausgelassen worden waren, selbst im als offiziell erfaßt bezeichneten Gebiet. Aber auch die Luftbildinterpretationsmethode zur Ortung und Bevölkerungszählung erforderte sehr sorgfältige Feldbeobachtungen und Tests. Nach der Überprüfung der beachtlich genauen Testresultate konnte die Methode auf die auch aus politischen Gründen von den offiziellen Zählbeamten gemiedenen Gebiete im Osten ausgedehnt werden. In offiziellen Schätzungen wurden bis zu 500000 Einwohner im Osten vermutet. Die Luftbildzählmethode ergab, abgestützt auf Bodenkontrollen, schließlich 95000 Einwohner.

Neben der in einigen Regionen zum ersten Mal kartographisch erfaßten administrativen Kleingliederung des Landes konnte der Bevölkerungszensus landesweit ausgedehnt und erstmals dargestellt werden. Durch die Kartierung und quantitative Darstellung der Bevölkerungsverteilung ist eine elementare Grundlage geschaffen worden, die für künftige Entwicklungs- und Versorgungsplanungen nicht nur im agrarischen Bereich dringend benötigt wird. Einerseits ist es wichtig zu wissen, daß die Bevölkerung 1975 offenbar 4,705 Millionen Einwohner umfaßte, und nicht wie zuvor geschätzt 5 bis 9 Millionen, und andrerseits ist die Tatsache zu wissen, wo und in welcher Anzahl und Dichte die Bevölkerung lebt, von entscheidender Bedeutung (STEFFEN et al, Lit. 5).

Damit ist ein erster Schritt in der Grunddatenbeschaffung getan. Die Luftbilder zeigen aber auch für den fast ausschließlich agrarisch genützten Raum, wo die Menschen arbeiten, was und wieviel angebaut und geerntet wird - somit, wie sie mit Grundnahrungsmitteln versorgt werden.

\section{Das Beispiel Nepal}

SCHMID (Lit. 6) hat in seiner Dissertation «Zur Wirtschaftsgeographie von Nepal» 1969 schon die gleiche Luftbildzählmethode versucht, doch ließ sie sich in diesem gänzlich anders strukturierten Raum nicht erfolgreich anwenden. Er mußte mit gemeindeweise erfolgten, z. T. persönlichen Schätzungen arbeiten. Dafür aber legte er durch eine sorgfältige Landnutzungskartierung vermittels Luftbildern in der Region Jiri, wo die Schweiz seit vielen Jahren an Aufbauarbeit beteiligt ist, den Grundstein für den ersten Agrarzensus.

Durch ausgedehnte Feldarbeit und den Vergleich von Landnutzungstypen mit ihrer Ausprägung im Luftbild erstellte er zunächst einen Luftbild-Inter- pretationsschlüssel. Dieser Schlüssel konnte dann verwendet werden, die Hauptbodenbedeckungstypen im Luftbild voneinander zu trennen und $\mathrm{zu}$ kartieren. In der so erstellten Landnutzungskarte wurden die Flächen der wichtigsten Agrarnutzungstypen ausplanimetriert, diese Zahlen mit mittleren Anbauflächenerträgen multipliziert und ein erster regionaler Ertragszensus damit ermöglicht.

Vermittels dieser relativ einfachen aber sehr effizienten Methode konnte SCHMID nachweisen, daß für eine gute Erntesaison die lokale Versorgung mit Grundnahrungsmitteln knapp ausreichte, daß aber bei der kleinsten Ernteeinbuße Versorgungsengpässe entstehen und die Einwohner hungern müssen.

Was hier in ein paar Sätzen Erwähnung findet basiert auf sehr intensiver Feldarbeit, der Erfragung und Analyse von Landnutzungs-, Anbau- und Erntezyklen, von Agrarpraktiken in ihren lokalen Ausprägungen usw. Ursachen und Wirkungen der sehr delikaten Gleichgewichtszustände, respektive der sich durch die zunehmende Bevölkerungszahl immer verschlechternden Versorgungslage, mußten analysiert werden. Selbstverständlich ist die Ernährungsund Versorgungslage damit nicht voll absehbar. SCHMID hat aber in minutiöser Kleinarbeit die Versorgungs- und Marktgewohnheiten der Einwohner sowie das Funktionieren der lokalen Märkte und ihrer Versorgung untersucht. Diese Marktanalysen, Verbrauchsermittlungen, Angebot- und NachfrageUntersuchungen sowie die Analyse des Warenverkehrs führten in Nepal zur zentralen Entwicklungsaufgabe, der Verbesserung der Verkehrsverbindungen.

SCHMID hat solche infrastrukturelle Untersuchungen 1967/68 durch Zählungen und Analysen durchgeführt, die bestehende Situation in Plandiagrammen und Statistiken dargestellt und Vorschläge zur besseren Verkehrsstrukturierung gemacht. Noch heute basiert ein Teil dieser wichtigen Aufbauarbeit, die von Bund und Helvetas getragen wird, auf diesen Basisuntersuchungen.

\section{Das Beispiel Liberia}

$\mathrm{KOCH}$ (Lit. 7) hat in Liberia die dortige Ausprägung und das Wirkungsgeflecht der Brandrodungskultur untersucht. Neben sehr intensiver Feldarbeit, die im tropischen Regenwald sehr mühsam und fast nur punktuell oder entlang von Routen erfolgen kann, verwendete er auch Luftbilder. Durch den Blick von oben und den Vergleich von Aufnahmen verschiedener Jahre gelang es, systematisch die Nutzungszyklen zu erfassen. Ergänzt hat er damals die zur Verfügung stehenden, für die Tropen wegen der Luftfeuchtigkeit typisch kontrastarmen Luftbildserien, durch von Hand aus einem Sportflugzeug aufgenommene Schräg- und Senkrechtbilder. 
Landnutzungskartierungen, die Erfassung dynamischer Veränderungen, Ernteschätzungen und die Ermittlung von Rotationszyklen werden in Kochs Dissertation beschrieben und bildeten wichtige Grundlagen zur Entwicklungsplanung und -Förderung.

Selbstverständlich bedurfte es außerdem sorgfältigster Feldbegehungen, sozio-ökonomischer und sozio-geographischer Untersuchungen. Dies ist natürlich kein Monopolanwendungsbereich für Geographen. Hier müssen, aufbauend auf den geschaffenen Grundlagen, der Agronom, der Wirtschaftswissenschafter, der Bodenkundler usw. eingesetzt werden, um letztlich Planer und Politiker mit geeigneten Entwicklungsstrategien und mit Grundlagen versorgen zu können.

\section{Die Datenlage}

Das Luftbild als billige Datenquelle für die verschiedensten Bedürfnisse wurde hier exemplarisch vorgestellt für Fälle, in denen die entsprechenden Informationen nicht anderweitig verfügbar waren. Zuerst muß diese Datenlage überprüft werden, da vielfach sehr ausführliche Untersuchungen bereits gemacht wurden und Statistiken verschiedenster Art existieren. Die Erhebung sozio-ökonomischer und soziologischer Grunddaten, z. B. durch Interviews und Fragebogentechniken, erfordert große lokale Sachvertrautheit und größtes Einfühlungsvermögen gegenüber der Bevölkerung, ihrer religiösen und kulturellen Erziehung und Einstellung.

In den letzten Jahren wurden immer mehr Satellitentechniken propagiert zur Kartierung und großräumigen Überwachung von natürlichen Hilfsgütern. Bekanntgeworden sind insbesondere die im LACIE (Large Area Crop Inventory Experiment) durchgeführten globalen Weizenernte-Prognosen und vielfältige Anwendungen im Agrarbereich in den Vereinigten Staaten.

Seit 1972 stützt man sich für diese Art von Untersuchungen ganz auf die multispektralen Bilder von Landsat. Auf einer sonnensynchronen polarnahen Umlaufbahn in ca. $920 \mathrm{~km}$ Höhe umkreist gegenwärtig Landsat-3 die Erde und nimmt mit zwei Abtastsystemen dabei $185 \mathrm{~km}$ breit streifenweise unseren Planeten auf. Alle 18 Tage ist eine globale Aufnahmesequenz fertig und der Satellit wieder über dem Ausgangspunkt. Je nach System beträgt das beste Auflösungsvermögen zwischen 45 und $79 \mathrm{~m}$. Gegenüber Luftbildern steht diese relativ geringe Auflösung als Nachteil an. Den Satellitenbildern gereicht aber zum großen Vorteil, daß synoptisch, d. h. zu einem Zeitpunkt, große Gebiete erfaßt werden ohne politische Grenzprobleme. Auch abgelegene Gebiete können erfaßt werden. Der größte Vorteil aber liegt darin, daß, wenn der Satellit einmal lanciert - er immer wieder periodisch vom gleichen
Gebiet Aufnahmen erstellen kann. Damit sind solche Meßdaten einerseits geeignet, großräumige Phänomene zu erfassen und andererseits deren dynamische Veränderungen festzustellen. Beispielsweise kann damit das Heranwachsen und Reifen von Getreide lokal oder global verfolgt, oder das weltweit gravierende Problem des Abholzens von tropischen Wäldern (s. Abb. 1 und 2) in seinen reellen Dimensionen erfaßt und unter Kontrolle gebracht werden.

\section{Das Beispiel Sri Lanka}

Auf Anfrage der Regierung von Sri Lanka an den Bund ist das Geographische Institut der Universität Zürich durch die DEH aufgefordert worden, das Sachwissen über die praktische Anwendbarkeit von Landsat-Aufnahmen zur Erhebung agrarischer Grunddaten in einem Entwicklungszusammenarbeits-Projekt einzusetzen. Dieses Projekt, das vor allem auf operationelle Reisernteprognosen ausgerichtet ist, eignet sich besonders, zu demonstrieren, daß es zwar einer enormen Technologie - hier auf amerikanischer Seite - bedarf, um einen Erderkundungssatelliten zu bauen und in Orbit zu bringen. Es zeigt aber auch, daß, wie im vorliegenden Falle, bei der weltweiten Verfügbarkeit von solchen Bildern auch erst noch zu günstigen Preisen - es wohl möglich und verantwortbar ist, unter Verwendung von demEntwicklungsland angepaßter Technologie diese Bilder nutzbringend zu verwerten.

Sri Lanka, an der Südspitze Indiens im Einflußbereich der regenbringenden Südwest- und NordostMonsune gelegen, weist im nordöstlichen Landesteil einen ausgeprägten Kontrast zwischen MonsunRegenzeit und Intermonsun-Trockenzeit auf. Während durch die nordöstlichen Strömungen im Winterhalbjahr meist genug Regen fällt, um eine sichere Reisernte auf dem gesamten bebaubaren Areal zu ermöglichen, so hängt eine zweite Aussaat und Ernte im trockenen Nordsommer ganz von künstlicher Bewässerung aus tausenden von kleineren und größeren Stauseen, sogenannten Tanks, ab.

Zuerst wurde versucht, das Bewässerungspotential für diese zweite Saison durch sorgfältige Ausmessung der Tanks zu errechnen und damit die zu erwartende Ernte zu schätzen. GEISER und vÖLKE, (Lit. 8) entwickelten auch eine sehr brauchbare Methode, die Wasservolumina zu berechnen. Aber nach einigen Tests mußte festgestellt werden, daß mit dem verfügbaren, kostbaren Wasser nicht immer ökonomisch umgegangen wird. Es werden beispielsweise die Felder bei Schädlingsbefall unter Zugabe von Chemikalien überflutet und so viel Wasser verschwendet. Andere Felder werden zwar bewässert aber nicht bebaut!

Damit kommen wir zur Grundfrage in diesem Projekt. Theoretisch reicht die mit Reis angebaute Flä- 
che zur Selbstversorgung der Bevölkerung Sri Lankas. Durch Variationen in Beginn und Dauer des Monsuns, durch Schädlingsbefall oder fehlendes Saatgut können aber sehr grosse Ertragsschwankungen auftreten. Zuverlässige Übersichten über das Ausmaß der Ernte sind jeweils ca. 2-3 Monate nach der Ernte in Colombo zentral verfügbar. Dann aber muß man bereits gegen die Auswirkungen z. B. einer Mißernte und dem damit verbundenen Hunger kämpfen. Dann werden die wenigen Devisen, die der Außenhandel und der Tourismus bringen eingesetzt, um zu horrenden Preisen Grundnahrungsmittel, vor allem Weizen und Reis, zu beschaffen. Vor drei Jahren war die Ernte so groß, daß man hoffte Lager anlegen zu können. Da die entsprechenden Einrichtungen nicht zeitgerecht zur Verfügung standen, verfaulte viel Getreide.

Aus solchen Gründen wurde im sogenannten «food crash»-Programm unsere Regierung angegangen, hoffend, daß Satellitentechniken eine frühere Schätzung der zu erwartenden Erträge ermöglichen werden.

Von GEISER und VÖLKE (Lit. 8 und 9) wurden entsprechende Methoden erarbeitet. Im SatellitenbildAusschnitt (siehe Abb. 1 und 2) sind neben Urwald, Grasland und Buschvegetation große zusammenhängende Reiskulturflächen abgebildet. Die Kartierung des bewässerten Reislandes hingegen war auch unter Zuhilfenahme modernster Computertechniken - die im Falle von Sri Lanka sowieso ungeeignet gewesen wären - nicht möglich. Ein indirekter Weg mußte gesucht werden.

Beim bewässerten Reis braucht es große Vorarbeiten, um die Felder nutzen zu können. Dadurch ist die Fläche, die man bei optimalem Wasservorrat bebauen kann, die sogenannte potentielle Anbaufläche, über mehrere Jahre hinweg konstant. Es galt, den Anteil der «tatsächlich bebauten Fläche» zu ermitteln. Zuerst wurde, unter Zuhilfenahme speziell aufbereiteter Satellitenaufnahmen und z.T. selbst hergestellter Luftbilder und mit viel Arbeitsaufwand im Feld, die potentielle Reisanbaufläche kartiert und eine sogenannte thematische Maske erzeugt, die mit Schwarz alles Nicht-Reisland abdeckend, über ein neues aktuelles Satellitenbild gelegt, nur noch die maximale Anbaufläche des Reises zeigt. Durch diese Maskierung wird es einfach, innerhalb dieser potentiellen Flächen die tatsächlich angebaute, sich durch einen Rotton (im Farbkomposit) abhebende Reisfläche, auszumessen.

Diese Flächendaten werden mit lokal vorhandenen, durchschnittlichen Erträgen pro Flächeneinheit multipliziert und in der operationellen Anwendung diese Ernteschätzungen der Regierung ca. einen Monat vor der Ernte zur Verfügung gestellt. Gegenwärtig laufen unter GEISER (Lit. 9 und 10) großangelegte Versuchsschätzungen und Genauigkeitsüberprüfungen.
Es wurde angedeutet, daß solche Satellitenbilder auch geeignet seien, längerfristige, großräumige Veränderungen zu erfassen. Im Sri-Lanka-Projekt konnte dies ebenfalls überprüft werden anhand einer sich zum weltweiten Problem herangesteigerten Praxis, dem Opfern riesiger tropischer Waldflächen für die Gewinnung neuen Ackerlandes. Es wird befürchtet, daß diese bedrohliche Entwicklung zur Verstärkung der Anreicherung der Atmosphäre mit $\mathrm{CO}^{2}$ beitragen wird. Die erwähnten Erderkundungsund Überwachungsmittel sind darum so wertvoll, weil z. B. die lokalen und globalen Ausmaße solcher Waldrodungen erfaßt werden können.

Abb. 1 zeigt einen Landsat-Ausschnitt von Ost Sri Lanka im Jahre 1973. Die dunkle Fläche im zentralen Teil des Bildes stellt eine Waldfläche von ca. $100 \mathrm{~km}^{2}$ dar. In Abb. 2 wird der gleiche Ausschnitt $61 / 2$ Jahre später gezeigt. Der größte Teil des Waldes ist bereits verschwunden!

Nicht nur die seit jeher auch hier ausgeübte Praxis der Brandrodung, die sich wegen der Bevölkerungsexplosion auch verstärkt hat, sondern insbesondere wegen riesigen international finanzierten Entwicklungsprojekten wurde ein großer Teil des ursprünglichen Waldes geopfert.

Zum Teil ist es zu spät, die Projekte zu verhindern. Es kann aber durch sorgfältige Untersuchungen der Veränderungen der ökologischen Systeme und Gleichgewichte vielleicht dazu beigetragen werden, daß die neuen Kulturflächen auch wirklich optimal genutzt, die Niederschlags-Einzugsbereiche geschützt, die Erosion durch entsprechende Maßnahmen reduziert werden. Es sind dies Herausforderungen nicht nur an den Geographen, sondern an die ganzen Naturwissenschaften, solche sich weltweit manifestierende Probleme lösen zu suchen.

\section{Ausblick}

Wenn wir zusammenfassen, so zeigt sich der Bereich Entwicklungszusammenarbeit als bedeutende Herausforderung an die Geographie. Erläutert wurden einige Beispiele von Grunddatenerhebungen mittels Befragungen, Feldarbeit, Karten, Luft- und Satellitenbildern, ohne Anspruch auf Vollständigkeit zu erheben. Innerhalb der Geographie sind es sämtliche Teilbereiche der Human- oder Anthropogeographie wie auch der physischen Geographie, die sich u. a. quantitativer Methoden und Hilfsmittel der Kartographie und der Fernerkundung sowie auch Methoden der Sozial- und Wirtschaftswissenschaften bedienen, um ein Optimum an Effizienz zu erreichen.

Hier steht die Geographie selbstverständlich nicht alleine dem Problem der Entwicklungszusammenarbeit gegenüber. Schließlich baut und installiert der Geograph keine Pumpen oder Staumauern, oder er 


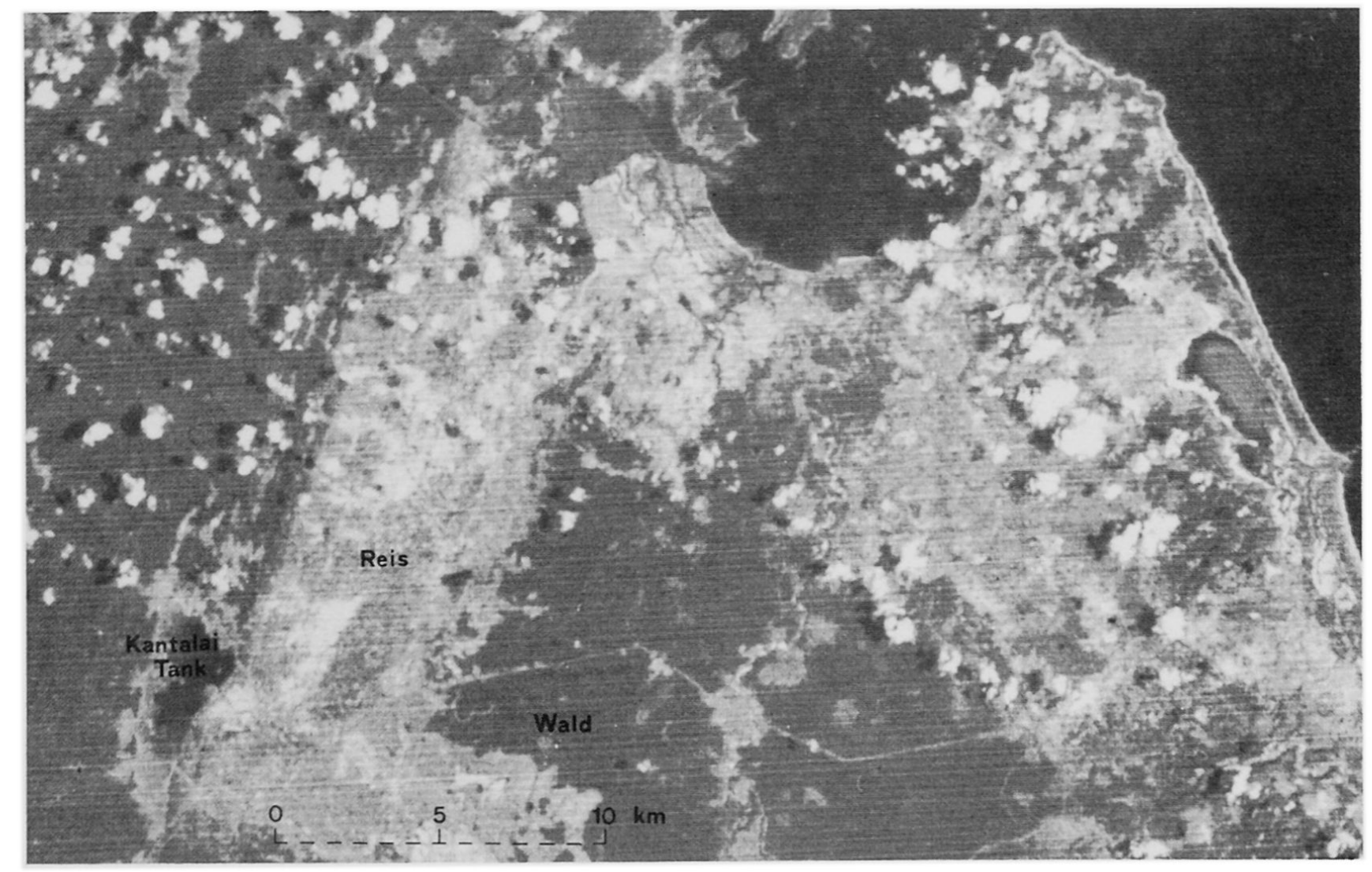

Abb. 1: Landsat-Bild (Kanal 5) vom 14. 3. 73. Region Kantalai an der Nordost-Küste Sri Lankas. Waldflächen in dunklem Grauton.

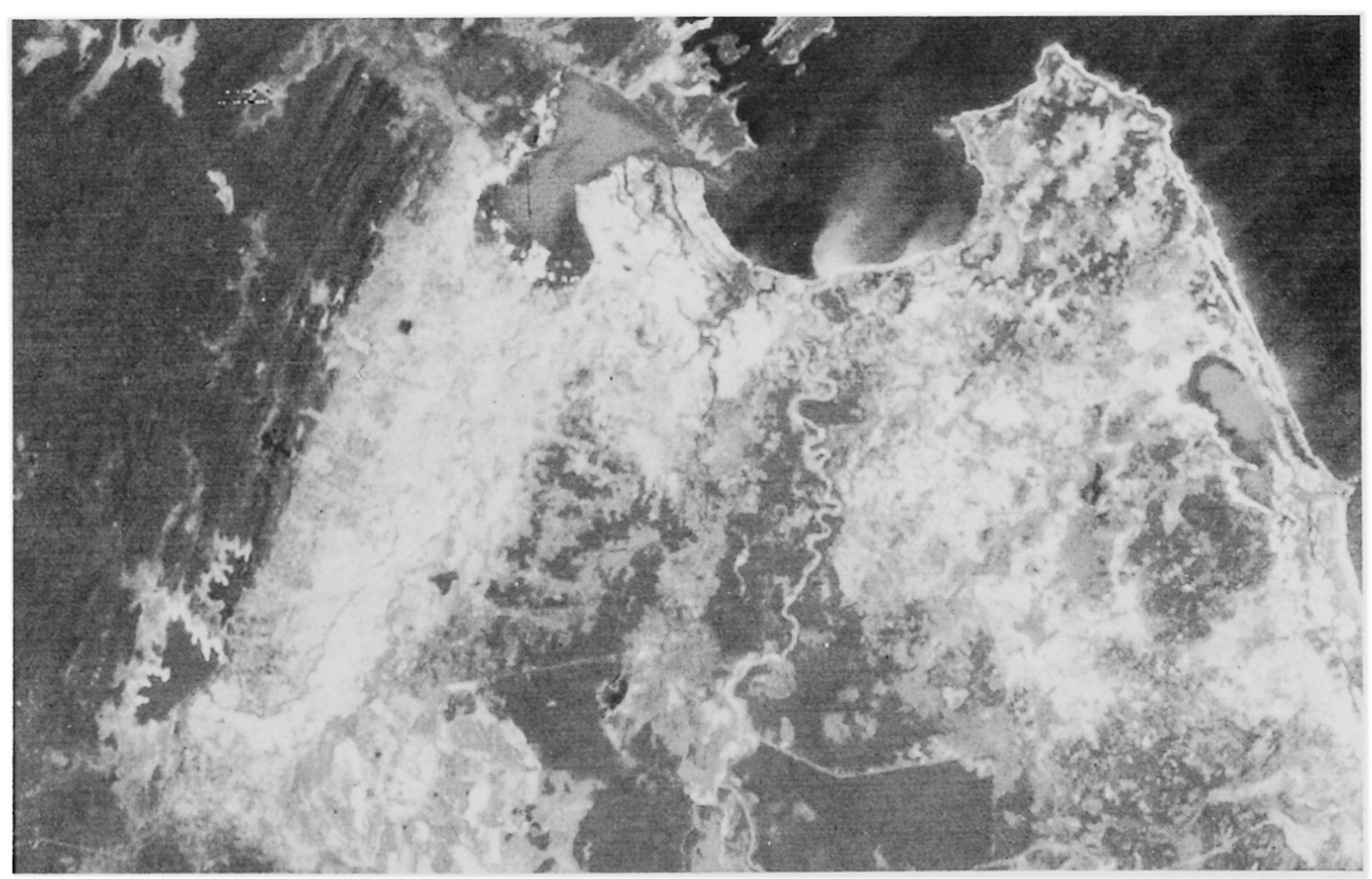

Abb. 2: Landsat-Bild (Kanal 5) vom 3.10.79 des gleichen Ausschnittes wie in Abb. 1. Gut erkennbar ist das Ausmaß der Waldrodungen in den $61 / 2$ Jahren. 
errichtet keine Viehzuchtfarmen. Er ist angewiesen auf die Fachbereiche der Natur- und Geisteswissenschaften, ebenso wie auf die Ingenieurwissenschaften.

Aber in den meisten Entwicklungszusammenarbeits-Projekten steckt ein zentral geographisches Potential, wie hier gezeigt an Beispielen zur Erhebung von Grund- und Systemdaten in Entwicklungsländern. Es ist zu hoffen, daß die Geographie dieses Potential weiterhin nutzen und ihre Möglichkeiten zum Nutzen der angesprochenen Partnerländer einsetzen werde.

\section{Literatur}

1) JOLLES, P.R.: Die Schweiz und der Nord-SüdDialog: Eine in den Realitäten verankerte Politik, in: Entwicklung/Développement No.1, p. 8-11, Bern 1979.

2) EDA: Kontrapunkt, in: Entwicklung/Développment No. 1, p. 26, Bern 1979.

3) TROLL, C.: Die Entwicklungsländer, ihre kulturund sozialgeographische Differenzierung, in: «Das Parlament» (Beil.) p. 791-800, Bonn, 28.12. 1960.
4) DOYLE, F. J.: Can Satellite Photography Contribute to Topographic Mapping, United Nations, World Cartography XII, p. 32-44, 1972.

5) STEFFEN, H. et al: Yemen Arab Republic - Final Report on the Airphoto Interpretation Project of the Swiss Technical Co-operation Service, Dept. of Geography, University of Zurich, Zurich 1978.

6) SCHMID, R.: Zur Wirtschaftsgeographie von Nepal, Dissertation Univ.Zürich, Juris Zürich 1969.

7) $\mathrm{KOCH}$, P.H.: Die Shifting Cultivation und ihre Luftbildinterpretation, Dissertation Univ. Zürich, Juris Zürich 1970.

8) GEISER, U. und vÖLKE, N.: Sri Lanka Rice Yield Forecasting with Landsat Imagery, Report on a Pilot Project, Dept. of Geography, University of Zurich, Zurich 1978.

9) GEISER, U.: Sri Lanka - Rice Yield Forecasting and Monitoring Land Use Changes Using Satellite Images, Primer Doc., Dept. of Geography, Univ. of Zurich, Zurich 1979.

10) GEISER U. und SOMMER, M.: The Sri Lanka/Swiss Satellite Imagery Interpretation Project, Report on the Testphase, Colombo 1981.

\section{Literaturbesprechung}

Kamerun, Bericht der Exkursion des Geogr.Instituts der Universität Bern. Geographica Bernensia G 9, Bern, 1978, 315 S., Fr. 43.--.

Wie eine anspruchsvolle Exkursion in ein mit der Schweiz seit langem in engerer Beziehung stehendes afrikanisches Land vorbereitet, durchgeführt, ausgewertet und das Resultat schliesslich Interessenten in überzeugender Form zugänglich gemacht werden kann, zeigt der vorliegende Band. In der Einleitung setzen die Autoren den Massstab:"Sinnvolle Vorbereitung und aufwendige Auswertung sind integrierende Bestandteile und geben der gezwungenermassen kurzen Exkursionszeit erst ihren Wert." (S.12)

Neun thematische Berichte vermitteln eine breite Einführung in Geographie, Wirtschaft, Geschichte und Kultur des Landes. Dass dabei die Entwicklung des Industriesektors und seine für das Land wesentlichen Probleme kaum Erwähnung finden, mag mit dem auf den Agrarsektor gelegten Schwerpunkt der Exkursion im Zusammenhang stehen, hinterlässt aber dennoch eine unbegründete Lücke. Das Erkennen komplexer Entwicklungszusammenhänge als Basis jeglicher Entwicklungszusanmenarbeit wird exemplarisch an neuen regionalen Spezialbe- richten versucht. Das bereitgelegte Grundlagenmaterial des ersten Teiles wird aufgegriffen, an Einzelbeispielen überprüft und mit Beobachtungen sowie Expertenmeinungen ergänzt. Besonders interessant ist der Abschnitt über die Spontansiedlungen in der Hafenstadt Douala und die beschriebene Aktivität der Selbsthilfe-Organisation in der "Zone Nylon", wenn auch die optimistischen Vermutungen über die künftige Entwicklung dieses Stadtquartiers in der Zwischenzeit vor allem aus politischen Gründen gedämpft werden müssen.

Im Synthesebericht wird versucht, die in den einzelnen Abschnitten erarbeiteten Fragen und Probleme der Entwicklungszusammenarbeit aufzugreifen, mit einem eigenen Grundschema von ökologisch-ökonomischen Systemen zu konfrontieren und in einen entwicklungspolitischen Rahmen zu stellen. Dieser anspruchsvolle Teil ist das Resultat der zweieinhalbtägigen Schlussbesprechung der Exkursion, die als Blockkurs durchgeführt wurde. Ein Exkursionstagebuch und organisatorische Hinweise schliessen den einheitlich gestalteten, mit Literaturangaben versehenen Bericht ab.

GH 2/81

Walter Rambousek 